\title{
SECOND CLASS CURRENTS AND MIRROR BETA DECAYS *
}

\author{
L. WOLFENSTEIN ** \\ Physics Department, University of Michigan, Ann Arbor, Michigan, USA \\ and \\ E. M. HENLEY \\ Physics Department, University of Washington, Seattle, Washington 98105, USA
}

Received 5 June 1971

\begin{abstract}
Answers are given to some questions raised by Lipkin concerning the relation between mirror asymmetry in beta-decay and second-class currents. Mirror asymmetry (not of electromagnetic origin) is evidence for second-class currents even taking into account possible $C P$ violation. A non-vanishing mirror asymmetry can be calculated in terms of single-nucleon transition matrices but there is no unique relation between the mirror asymmetry and the matrix elements of the second-class current for free nucleons.
\end{abstract}

A recent analysis by Wilkinson [1] and experiments by Wilkinson and Alburger [2] concerning mirror beta-decays have revived interest in the question of the existence of secondclass currents. Such currents. first discussed by Weinberg [3]. have opposite signs under the G transformation from the "normal" strangeness-conserving currents included in the standard treatments of weak interactions.

The presence of second-class currents can be observed in a variety of ways $[3,4]$. In this note we restrict ourselves to "mirror asymmetry". by which we mean a difference in the ft-values of mirror decays which cannot be explained by electromagnetic corrections 4 . Recently Lipkin [5] has raised some questions concerning the relevance of any observed mirror asymmetry to the existence of second-class currents. It is the purpose of this letter to give at least partial answers to these questions.

Is a mirror asymmetry evidence for secondclass currents even when $C P$ violation is taken into account? We assume $C P T$ invariance. The answer is then clearly yes. This result follows from theorem 1 of Weinberg's original paper [3] which takes $C P$ violation into account. The theorem states \# that, assuming $C P T$ invar-

* Supported in part by the US Atomic Energy Commission.

** On leave from Carnegie-Mellon University. Pittsburgh, Pennsylvania, USA. iance and neglecting the final-state electromagnetic interactions, one finds in going from the strangeness-conserving decay

$A \rightarrow B+l^{-}+i$

to the mirror decay

$A^{\mathrm{M}} \rightarrow B^{\mathrm{M}}+l^{+}+\nu$,

that the following relations hold:

1) For $P T$-even observables terms in $V_{I} \cdot A_{I}$, $A_{\mathrm{I}} \cdot A_{\mathrm{II}}, V_{\mathrm{I}} \cdot V_{\mathrm{II}}$ and $V_{\mathrm{II}} \cdot A_{\mathrm{II}}$ change sign; others do not.

2) For $P T$-odd observables terms in $V_{\mathrm{I}} \cdot A_{\mathrm{I}}$, $A_{\mathrm{I}} \cdot A_{\mathrm{II}}, V_{\mathrm{I}} \cdot V_{\mathrm{II}}$ and $V_{\mathrm{II}} \cdot A_{\mathrm{II}}$ do not change sign; others do.

Here A, B are hadrons; $l, \nu, \bar{\nu}$ are leptons and the mirror state $|A M\rangle$ is related to $A$ by

\$ We do not discuss whether the present observations as summarized by Wilkinson [1] indicate the existence of such a mirror asymmetry or can aiternatively be explained as due to electromagnetic effects.

\# Weinberg's statement of the theorem is somewhat ambiguous, but the conclusions of the theorem are not; our formulation is the same as that of Cabibbo [6] except that he uses the $U$ transformation rather than $G$ to distinguish first- and second-class currents. The theorem holds for any mirror pair which take part in semi-leptonic processes, e.g., neutrino reactions.

I A PT-odd observable is one which violates parity but not time-reversal invariance, as $\langle\boldsymbol{\sigma} \cdot \boldsymbol{p}\rangle$, or one which violates time-reversal but not parity invariance, as $\left\langle\boldsymbol{\sigma} \cdot \boldsymbol{p}_{1} \times \boldsymbol{p}_{2}\right\rangle$. 


$$
\left|A^{\mathrm{M}}\right\rangle=\exp \left(\mathrm{i} \pi T_{2}\right)|A\rangle \equiv U|A\rangle
$$

where $T_{2}$ is the 2-component of the isospin operator. The first-and second-class axial and vector currents are distinguished by

$$
\begin{aligned}
& G V_{\mathrm{I}(\mathrm{II})} G^{-1}=+(-) V_{\mathrm{I}(\mathrm{II})}, \\
& G A_{\mathrm{I}(\mathrm{II})} G^{-1}=-(+) A_{\mathrm{I}(\mathrm{II})} .
\end{aligned}
$$

Since the rate of a decay or of a reaction is a $P T$-even observable and no $V-A$ interference terms appear in decay rates [6]. it follows that any mirror asymmetry must arise from $V_{\mathrm{I}} \cdot V_{\mathrm{II}}$ or $A_{\mathrm{I}} \cdot A_{\mathrm{II}}$. Of course, if the second-class current term were entirely odd under $C P$, its inter ference with a $C P$-conserving first-class current could not be detected by a measurement of a rate but would require a measurement of a $T$-odd observable. This possibility has been analyzed in detail by Kim and Primakoff [7].

Can a non-vanishing mirror asymmetry be calculated for single-nucleon transitions? If second-class currents exist, then there is no reason for ruling out second-class form factors in single-nucleon transitions. However, Lipkin argues that, even in the presence of secondclass form factors, hermiticity guarantees the equality of the rates $\mathrm{n} \rightarrow \mathrm{p}+\mathrm{e}^{-}+\bar{\nu}$ and $\mathrm{p}-\mathrm{n}+\mathrm{e}^{+}+\nu$. We show below by an explicit relativistic calculation that a mirror asymmetry can be calculated for a single-nucleon transition and that Lipkin's argument fails when the nucleons are not on -the-mass shell. Our conclusion is in agreement with explicit nuclear calculations using non-relativistic single-particle operators $[8,9]^{\dagger}$.

For the axial current, to which we now limit ourselves, the single-nucleon matrix element may be written $\#$

† It should be emphasized that we do not intend our relativistic off-mass-shell calculation to be taken as a serious method of calculating nuclear transitions. Explicit calculations that depend on the off-massshell character of nucleons are likely to be ambiguous or model-dependent because the properties of such nucleons really depend upon the nature of the interaction which takes them off-mass shell. Oux purpose rather is to gain insight into the apparent contradiction between Lipkin's conclusion and the nuclear calculations.

H Our notation is that of ref. [10] except that we use $J_{\mu}^{*}$ (rather than $\bar{J}_{\mu}$ ). where $J_{i}^{*}=J_{i}^{\dagger}, J_{4}^{*}=-J_{4}^{\dagger}$. In this notation $\gamma_{\mu}$ is hermitian and $A \cdot B=A_{\mu} B_{\mu}=\boldsymbol{A} \cdot \boldsymbol{B}$ $A_{4} B_{4}$.

$$
\begin{aligned}
& \left\langle\mathrm{p}\left(p^{\prime}\right)\left|A_{\mu}\right| \mathrm{n}(p)\right\rangle \\
& \quad=\bar{u}_{\mathrm{p}}\left(p^{\prime}\right)\left[g_{\mathrm{A}} \gamma_{\mu} \gamma_{5}+\mathrm{i} f_{\mathrm{A}^{\gamma} 5} q_{\mu}-h_{\left.\mathrm{A}^{\gamma} 5^{\sigma}{ }_{\mu \nu} q_{\nu}\right] u_{\mathrm{n}}(p)}\right.
\end{aligned}
$$

where $q=p^{\prime}-p$, and $g_{\mathrm{A}}, f_{\mathrm{A}}, h_{\mathrm{A}}$ are form factors which are functions of the scalars $p^{\prime 2}, p^{2}$. $q^{2}$. On the mass shell this dependence reduces to one on $q^{2}$ alone. Invariance of the whole Hamiltonian under $C P$ or $T$ leads to the restriction that the form factors are real. First-class form factors satisfy the relations

$$
\begin{aligned}
& g_{\mathrm{AI}}\left(p^{2}, p^{2}, q^{2}\right)=g_{\mathrm{AI}}\left(p^{2}, p^{2}, q^{2}\right) \\
& f_{\mathrm{AI}}\left(p^{2}, p^{2}, q^{2}\right)=f_{\mathrm{AI}}\left(p^{2}, p^{2} \cdot q^{2}\right) \\
& h_{\mathrm{AI}}\left(p^{\prime 2}, p^{2}, q^{2}\right)=-h_{\mathrm{AI}}\left(p^{2}, p^{\prime 2}, q^{2}\right)
\end{aligned}
$$

whereas the sign on the right-hand side is opposite for second-class form factors. On the mass shell, $p^{\prime 2}=p^{2}=M^{2}$, if we neglect the electromagnetic neutron-proton mass difference, so that $h_{\mathrm{A}}$ must be a second-class form factor while $g_{\mathrm{A}}$ and $f_{\mathrm{A}}$ are the usual first-class form factors. If we neglect the variation in the form factors with $\left(p^{2}-p^{2}\right)$, then it follows from the theorem quoted above that any mirror asymmetry in beta-decay must be due to an interference between $g_{\mathrm{A}}$ and $h_{\mathrm{A}} .\left(f_{\mathrm{A}}\right.$ is unimportant in nuclear beta-decay.) The importance of a possible dependence on $\left(p^{2}-p^{2}\right)$ is discussed later.

The decay rate $\mathcal{R}$ is proportional to the square of the matrix element averaged over initial spins and summed over final spins and over the internal angle variables of the leptons for fixed $q_{\mu}$. For fixed values of $p$ and $p^{\prime}$

$\mathcal{R}=\bar{N}_{\mu \nu}\left[A\left(q^{2}\right) q_{\mu} q_{\nu}+B\left(q^{2}\right) \delta_{\mu \nu}\right]$.

where the square bracket is the lepton contribution and $\bar{N}_{\mu \nu}$ comes irom the hadron current [10]. An explicit calculation using eq. (3) gives for the interference term in $\bar{N}$

$N_{\mu \nu} \sim 2 M \operatorname{Re}\left(g_{\mathrm{A}} h_{\mathrm{A}}^{*}\right)\left[P_{\mu} q_{\nu}+P_{\nu} q_{\mu}-2 \delta_{\mu \nu} P \cdot q\right]$,

where $P=\left(p+p^{\prime}\right)$. Thus, the interference contribution, $I$, to the rate $\mathcal{R}$ is

$I \sim-12 M \operatorname{Re}\left(g_{\mathrm{A}} h_{\mathrm{A}}^{*}\right) P \cdot q B\left(q^{2}\right)$.

Lipkin's assertion that the interference term must vanish because of a $90^{\circ}$ phase difference is not the case unless the additional assumption is made that $h_{\mathrm{A}}$ is purely imaginary (that is, $C P$ odd) or, equivalently [11], $U A_{\mu} U^{-1}=-A_{\mu}^{*}$. 
However, $I$ does vanish on the mass shell if we neglect the electromagnetic mass difference since

$P \cdot q=p^{2}-p^{2}=\left(M_{\mathrm{n}}^{2}-M_{\mathrm{p}}^{2}\right) \approx 0$.

In complex nuclear beta-decays the nucleons are not on-the-mass shell and using the standard non-relativistic approximation

$P \cdot q \approx 2 M\left(E_{l}+E_{\nu}\right)$

one readily notes that $I$ does not vanish. Lipkin's argument based on hermiticity works when one compares the energetically-possible $\mathrm{n} \rightarrow \mathrm{p}+\mathrm{e}^{-}+\bar{\nu}$ with the impossible $\mathrm{p} \rightarrow \mathrm{n}+\mathrm{e}^{+}+\nu$ both involving nucleons (but neglecting electromagnetic mass differences in the calculation on-the-mass shell). In mirror transitions in nuclei, however, we are comparing the transition "loosely-bound neutron to tightly-bound proton" with "loosely-bound proton to tightly bound neutron".

Hermiticity gives

$\left\langle\mathrm{p}\left(p^{\prime}\right)\left|A_{\mu}\right| \mathrm{n}(p)\right\rangle=\left\langle\mathrm{n}(p)\left|A_{\mu}^{\dagger}\right| \mathrm{p}\left(p^{\prime}\right)\right\rangle^{*}$,

so that by going to the Breit frame and making a suitable rotation or reflection one obtains [5]

$$
\begin{aligned}
& \left\langle\mathbf{p}\left(\frac{1}{2} \boldsymbol{q}, E^{\prime}\right)\left|A_{\mu}\right| \mathrm{n}\left(-\frac{1}{2} \boldsymbol{q}, E\right)\right\rangle= \\
& \quad \pm\left\langle\mathrm{n}\left(\frac{1}{2} \boldsymbol{q}, E\right)\left|A_{\mu}^{*}\right| \mathbf{p}\left(-\frac{1}{2} \boldsymbol{q}, E^{\prime}\right)\right\rangle^{*}
\end{aligned}
$$

Although the initial and final state momenta are the same on both sides of eq. (9), the energies differ by $E \leftrightarrow E^{\prime}$, unless the matrix element is taken between states on the mass shell. Lipkin omits the energy dependence; his argument therefore fails to the extent that one or both nucleons are off the mass shell.

Given nuclear wave-functions, is it possible to relate uniquely the matrix element of the second-class current for free nucleons to an observed mirror asymmetry? We show below by an explicit construction that the relation is ambiguous because of the intrinsic off-mass-shell character of the mirror asymmetry calculation. For this reason it could be said that the "impulse approximation" is not applicable to the calculation of mirror asymmetries.

An alternative form to eq. (3) for the matrix element is

$$
\begin{aligned}
& \left\langle\mathrm{p}\left(p^{\prime}\right)\left|A_{\mu}\right| \mathrm{n}(p)\right\rangle=\overline{\mathrm{u}}\left(p^{\prime}\right)\left[g_{\mathrm{A}}^{\prime} \gamma_{\mu} \gamma_{5}\right. \\
& \left.+\mathrm{i} f_{\mathrm{A}} \gamma_{5} q_{\mu}+\mathrm{i} \bar{h}_{\mathrm{A}}^{\prime} \gamma_{5} P_{\mu}\right] u_{\mathrm{n}}(p) .
\end{aligned}
$$

The two forms are equivalent on the mass shell if $[10]$
$h_{\mathrm{A}}^{\prime}=h_{\mathrm{A}}, \quad g_{\mathrm{A}}^{\prime}=g_{\mathrm{A}}-\left(M_{\mathrm{n}}-M_{\mathrm{p}}\right) h_{\mathrm{A}} \approx g_{\mathrm{A}}$.

The alternatives to eqs. (6) and (7) are

$N_{\mu \nu}^{\prime} \approx 2 M \operatorname{Re}\left(g_{\mathrm{A}} h_{\mathrm{A}}^{\prime *}\right)\left(P_{\mu} q_{\nu}+P_{\nu} q_{\mu}\right)$

$I^{*} \approx 4 M \operatorname{Re}\left(g_{\mathrm{A}} h_{\mathrm{A}}^{\prime *}\right) P \cdot q\left\{A\left(q^{2}\right) q^{2}+B\left(q^{2}\right)\right\}$.

Thus the interference term is completely different in terms of $h_{\mathrm{A}}^{\prime}$ as compared to $h_{\mathrm{A}}$. This point has already been made for the corresponding nuclear calculation [9].

The source of this difference, in our formulation, is the possibility of a matrix element of the axial current of the form

$\left(p^{\prime 2}-p^{2)} h_{\mathrm{A}}^{\prime \prime}\left(q^{2}\right) \bar{u}_{\mathrm{p}}\left(p^{\prime}\right) \gamma_{\mu} \gamma_{5} u_{\mathrm{n}}(p)\right.$.

It follows from eq. (4a) that this is a secondclass form factor; it corresponds to a term in the expansion of $g_{\mathrm{A}}\left(p^{2}, p^{2}, q^{2}\right)$ that is odd in $\left(p^{2}-p^{2}\right)$. On the mass shell this term is just equal to the relatively unimportant difference between $g_{A}^{\prime}$ and $g_{A}$ noted in eq. (10). but it can be important off the mass shell. It is the possibility of such a form factor or, equivalently, the unknown dependence on $\left(p^{2}-p^{2}\right)$ of the form factors $g_{\mathrm{A}}$ and $h_{\mathrm{A}}$, which makes it impossible to relate an observed nuclear mirror asymmetry to a nucleon matrix element.

It should be noted that even if it is assumed that the form factors are independent of $\left(p^{\prime 2}-p^{2}\right)$ the mirror asymmetry is still dependent on the fact that the nucleons are not on the mass shell because of the "kinematic factor" $P \cdot q$ in eq. (7) or ( $\left.7^{\prime}\right)$. However, it follows from the discussion above that even if it is assumed that $g_{\mathrm{A}}$ is independent of $\left(p^{2}-p^{2}\right)$ this independence does not hold for $g_{\mathrm{A}}^{\prime}$. Thus it is not possible to distinguish the effect of a form factor dependence on $\left(p^{2}-p^{2}\right)$ from the "kinematic factor".

Should the mirror asymmetry be proportional to the energy release? It follows from eqs. (7) and (8) as well as from nuclear calculations $[8,9]$ that the single-nucleon contribution to mirror asymmetry is proportional to the energy release (i.e., it vanishes as this energy goes to zero). Indeed, if we neglect electromagnetic mass differences, the energy release is just a measure of the extent to which the single-nucleon transition is off mass shell. However, contributions that cannot be described in terms of single nucleon matrix elements, such as meson-exchange effects, are not necessarily proportional to the energy difference of parent and daughter nuclei [9]. The answer to the question posed is 
thus model dependent. There are two reasons for questioning the predominance of the single nucleon contribution. As pointed out by Weinberg [3], the single nucleon contribution is intrinsically small just because of its proportionality to the energy release. The other point, emphasized by Lipkin, is that it is incorrect to treat the effects of binding by a one-particle approximation, since the binding is due to the other nucleons in the nucleus and so, in part, is a many-body effect.

One of the authors (EMH) would like to thank Professor Denys Wilkinson for numerous stimulating discussions on the subject of secondclass currents. Both of us wish to thank Professor Harry Lipkin for private communications on this subject.

\section{References}

[1] D. H. Wilkinson, Phys. Letters $31 \mathrm{~B}$ (1970) 447.
[2] D.H.Wilkinson and D. E. Alburger, Phys. Rev. Letters 24 (1970) 1134; 26 (1971) 1127: Phys. Letters 32B (1970) 190 .

[3] s.Weinberg, Phys. Rev. 112 (1958) 1375.

[4] C.W.Kim, Phys. Letters 34B (1971) 383; B.R.Holstein and S.B.Treiman, Phys. Rev., to be published.

[5] H.J.Lipkin, Phys. Letters 34B (1971) 202; Mirror asymmetry for pedestrians (unpublished).

[6] N. Cabibbo. in: Particle symmetries, Vol. II, Brandeis University Summer Institute in Theoretical Physics, 1965 (Gordon and Breach. 1966) p.1.

[7] C.W.Kim and H. Primakoff, Phys. Rev. 180 (1969) 1502 .

[8] J.N.Huffaker and E.Greuling, Phys. Rev. 132 (1963) 738; R.J.Blin-Stoyle and M.Rosina, Nucl. Phys. 70 (1965) 321 .

[9] J. Delorme and M. Rho. Phys. Letters 34B (1971) 238.

[10] R.E. Marshak. Riazuddin and C. P. Ryan, Theory of weak interactions in particle physics (Wiley and Co.. 1969) pp. $314 \mathrm{ff}$.

[11] J.Bernstein, Elementary particles and their currents (W. H. Freeman and Co.. 1968) Ch. 9. 